\title{
Performance evaluation of cooperative ML-IDMA communications with DF protocol
}

\author{
Basma A. Mahmoud, Esam A. A. Hagras, Mohamed A. Abo El-Dhab \\ Department of Electronics \& Communications, Arab Academy for Science, Technology \& Maritime Transport, Cairo, Egypt
}

\section{Email address:}

basmaamahmoud@yahoo.com(B. A. Mahmoud), esamhagras_2006@yahoo.com(E. A. A. Hagras), mdahab@aast.edu(M. A. A. El-Dhab)

\section{To cite this article:}

Basma A. Mahmoud, Esam A. A. Hagras, Mohamed A. Abo El-Dhab. Performance Evaluation of Cooperative ML-IDMA Communications with DF Protocols. American Journal of Networks and Communications. Vol. 2, No. 3, 2013, pp. 56-61. doi: 10.11648/j.ajnc.20130203.11

\begin{abstract}
Relaying and cooperative diversity allow multiple wireless radios to effectively share their antennas and create a virtual antenna array, thereby leveraging the spatial diversity benefits of multiple-input multiple-output antenna systems. In this paper, we consider the bit error rate performance analysis of a cooperative relay communication system on MultiLayer-IDMA (ML-IDMA) using Maximal-Ratio-Combining (MRC) technique; we examine the effect of layers number on the performance and derive the average bit error probability of the Decode-and-Forward (DF) relay schemes by using the closed-form relay link Signal-to-Noise Ratio (SNR). Based on the analysis, we show that from the simulation results, degradation in the performance is observed when increasing number of layers but on the other hand we saved in the Band Width (BW).
\end{abstract}

Keywords: Cooperative Transmission, Multi Layers, Mrc, Df

\section{Introduction}

Multiple-Inputs Multiple-Output (MIMO) technology is widely used due to its ability to offer high diversity and multiplexing gain. The Impracticality of mounting multiple antennas on a mobile device favor other techniques to be used in wireless communication and these techniques are also designed to have less impact on the size and power consumption of the devices. Recently, it has been shown that, in a cooperative system, two or more users cooperate with each other to transmit information to the destination. The cooperative users can share each other's antennas to form a virtual multiple antenna system so that a single antenna device can also benefit from the spatial diversity provided by the cooperative users. In such a way, cooperative communication allows a source node with a single antenna to share the antennas of other nodes, resulting in a form of virtual MIMO system[1].

User cooperation systems that utilize different cooperative signaling methods are known to improve cellular system capacity and coverage. The relay node physical limitation and allowed signaling complexity are the two criteria that limit the used cooperation system. $\operatorname{In}[2$, 3] several cooperative diversity protocols were developed and analyzed; Amplify-and-Forward (AF), Decode-andForward (DF), Detect-and-Forward (DtF), Estimate-andForward (EF) and Selective-DF (S-DF).

In AF protocol, relay nodes amplify the signals received from a source node and transmit the amplified version of the signals to a destination node. In EF, the relays forward an estimate of its received signals to the destination .For $\mathrm{DtF}$, the relays detect the received signals and forward the detected symbols to the destination. For DF, the relay nodes decode the information received from the source and re-encode the signal before transmitting it to the destination. For S-DF, only those nodes that can correctly decode are selected to forward the signals to the destination[4].

In such a way, cooperative communication allows a source node with a single antenna to share the antennas of other nodes, resulting in a form of virtual multiple-input multiple-output (MIMO) system.

As a kind of non-orthogonal multiple access scheme, Interleave Division Multiple Access (IDMA) has been widely researched[5], which is regarded as a special form of Code Division Multiple Access (CDMA) by treating interleaving index sequences as multiple access codes. IDMA performance is better than the conventional CDMA regarding the power and bandwidth efficiency. IDMA has 
common advantages with CDMA, diversity against fading and mitigation of the user interference problem, are two important ones. A low-cost turbo-type Multi-User Detection (MUD) algorithm applicable to the system with large numbers of users, which is crucial for high-rate multiple access communication, is an important unique advantage of the IDMA that can add to its bandwidth efficiency as well as high transmission speed of data[6].

The principle of the IDMA systems is that the chip-level interleavers should be different for different users. In addition, a low-cost chip-by-chip iterative detection scheme can be utilized in the IDMA systems. Motivated by the concept of IDMA, Superposition Coded Modulation (SCM) partitions the data to multi layer, where each layer is treated by a user equivalently[7]. Multi-layer IDMA (ML-IDMA) is a special form of superposition coding scheme and it can be considered as a joint modulation/channel coding transmission scheme. Based on these backgrounds, according to the above observations, we propose a cooperative transmission scheme based on ML-IDMA.

In[6, 9-11], authors' have paid attention to the one layer IDMA cooperative system and without using any combiner technique at the destination. In this paper, we carry out the performance analysis of cooperative single user ML-IDMA scheme for equidistant relaying geometry with different number of layers and a Maximal-Ratio-Combining (MRC) technique; relay protocol that is used in this paper is Decode-and-Forward (DF). The rest of the paper is organized as follows: in Section 2, we discuss the MLIDMA system with DF Protocol; the experimental result is presented in Section 3; while conclusions and future works are presented in Section 4.

\section{ML-IDMA System with DF Protocol}

Recently a wireless transmission is very seldom analogue and the relay has enough computing power, so DF is most often the preferred method to process the data in the relay. The received signal is first decoded and then re-encoded. So there is no amplified noise in the sent signal, as is the case using AF protocol.

In the case of IDMA with DF, the original data is extracted from the received signal by using iterative MultiUser Detection (MUD) at each relay. Decoded data is further encoded by the relay in the similar way as encoded by the source. Encoded signal is finally sent to the destination. In literature, DF is regenerative process, so it can eliminate the Source-Relay (S-R) noise from the interuser signal. At low SNR if the decoding at the relay is unsuccessful then the performance of DF will be degraded[12].

We consider the cooperative system in Figs. (1-4). the system consists of a source (S), (M) relays, and a destination (D). The signal is transmitted through two phases. For the first phase the source broadcasts the signal to the relays, and the relays transmit their signals for the second phase to the destination.

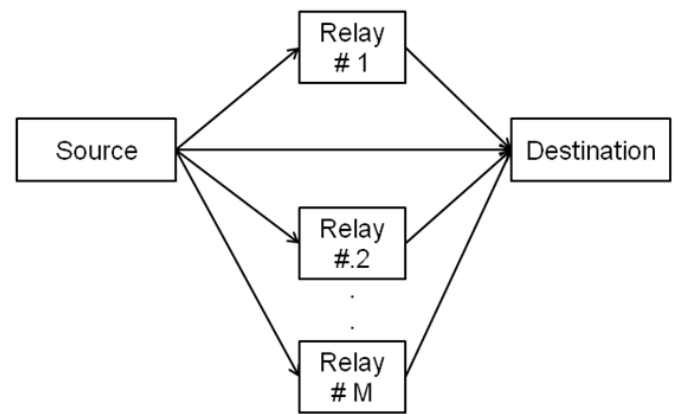

Figure 1. System model for cooperative ML-IDMA relay system with multiple relays.

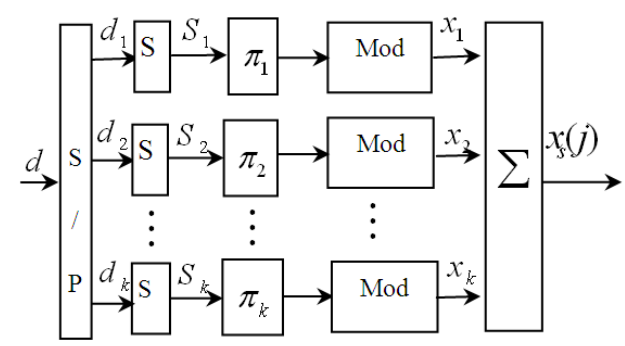

Figure 2. ML-IDMA transmitter (Source)

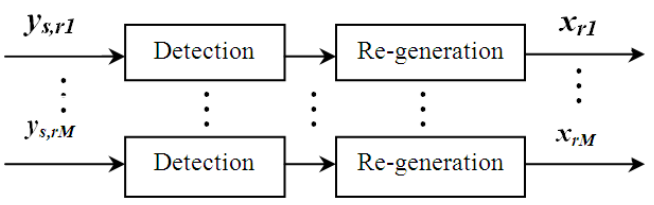

Figure 3. Relays with Decode-and-forward protocol
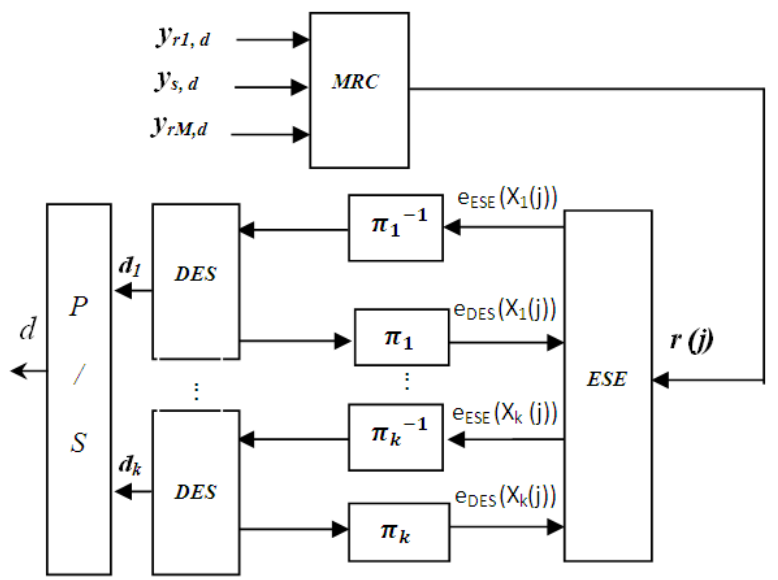

Figure 4. ML-IDMA Receiver (Destination)

In our design, the source/user generates an input data sequence $d=[d(1), d(2) \ldots, d(N)]$ which is converted by serial-to-parallel converter into $K$ subsequences layers. Then the data, in each layer, is spread, interleaved and modulated, independently. Finally, all data in $K$ layers are linearly superimposed to transmission then the source broadcasts the superimposed signal to the relays.

For $K$-layer, the data sequence $d_{k}=\left[d_{k}(1), d_{k}(2) \ldots, d_{k}\right.$ (I)] is first spread, generating a spread sequence $s_{k}=\left[s_{k}(1)\right.$, $\left.s_{k}(2) \ldots, s_{k}(J)\right]$ then the spread sequence $s_{k}$ is interleaved by a distinct chip-level interleaver $\pi_{k}$ to produce a 
permutated sequence $s_{k}$. After interleaving, the randomly sequence $s_{k}$ is modulated to $x_{k}=\left[x_{k}(1), x_{k}(2) \ldots, x_{k}(J)\right]$ by BPSK. Where $I=N / K, N$ is the user data length and I is the layer data length.

The transmitted signal $x_{s}$, after sum mission from $K$ Layers, is then given by:

$$
x_{s}(j)=\sum_{k=1}^{K} x_{k}(j) \quad j=0,1 \ldots, J
$$

By assuming that each terminal has one antenna; each relay decode/detect the received signal and retransmit it after regeneration of the decoded/detected signal to the destination. Assume that the channel between the source and each relay is a quasi-static Rayleigh fading channel with Additive White Gaussian Noise (AWGN).

The received signal at the relay is given by:

$$
y_{s, r}=\sqrt{P_{t}} h_{s, r} x_{s}+n_{s, r}
$$

where, $h_{s, r}$ is the channel coefficient between the source and relay, and $n_{s, r}$ is a sample of an AWGN process with zero mean and variance $\sigma 2$ per dimension between source and relay.

By normalizing the power to $\mathrm{P}_{\mathrm{t}}=1, \mathrm{y}_{\mathrm{s}, \mathrm{r}}$ is given by:

$$
y_{s, r}=h_{s, r} x_{s}+n_{s, r}
$$

We will represent the signal at the relay after detection and regeneration as $x_{r}$. Where the detection algorithm that is done at the relay using DF protocol was the same as done at the destination (will be discussed later).

The received signal at the destination at the first phase is given by:

$$
\mathrm{y}_{\mathrm{s}, \mathrm{d}}=h_{S, d} x_{s}+n_{s, d}
$$

where, $h_{s, d}$ is the channel coefficient between source and destination, and $n_{s, d}$ is a sample of an AWGN process with zero mean and variance $\sigma 2$ per dimension between source and destination.

The relayed signal when received at destination is given by:

$$
y_{r, d}=h_{r, d} x_{r}+n_{r, d}
$$

where, $h_{r, d}$ is the channel coefficient between relay and destination, and $n_{r, d}$ is a sample of an AWGN process with zero mean and variance $\sigma 2$ per dimension between relay and destination.

After receiving the relayed signal $\left(y_{r, d}\right)$ and direct signal $\left(\mathrm{y}_{\mathrm{s}, \mathrm{d}}\right)$ from relay and source respectively, the total received signal (r (j)) at the destination for Maximum-RatioCombining (MRC) receiver is given by:

$$
r=h_{s, d}^{*} \mathrm{y}_{\mathrm{s}, \mathrm{d}}+\sum_{\mathrm{m}=1}^{\mathrm{M}}\left(h_{s, r_{m}}^{*} * h_{r_{m}, d}^{*}\right) \mathrm{y}_{\mathrm{r}_{\mathrm{m}}, \mathrm{d}}
$$

where, $\mathrm{M}$ is the total number of relays.

Using a single-path / one relay system $(\mathrm{M}=1)$, we can rewrite Eq. (6) as:

$$
\begin{gathered}
r=\left(h_{s, r}^{*} * h_{r, d}^{*}\right) \mathrm{y}_{\mathrm{r}, \mathrm{d}} 8 \\
r=\left(h_{s, r}^{*} * h_{r, d}^{*}\right) h_{r, d} x_{r}+\left(h_{s, r}^{*} * h_{r, d}^{*}\right) n_{r, d} \\
r(j)=h \sum_{k=1}^{K} x_{k}(\mathrm{j})+n(\mathrm{j})
\end{gathered}
$$

where, $h=\left(h_{s, r}^{*} * h_{r, d}^{*}\right) h_{r, d}, \quad n=\left(h_{s, r}^{*} * h_{r, d}^{*}\right) n_{r, d}$ represents the composite noise.

Since each layer of ML-IDMA systems can be treated as one user of multi-user systems, the suboptimal iterative detection[13] and [ 14] can be applied in ML-IDMA systems. The receiver of ML-IDMA is illustrated in Fig. 4, which is composed by an Elementary Signal Estimator (ESE) and $K$ de-spreaders (DESs). They are applied to solve inter-layer interference and the spreading constraint separately $[15,16]$. The receiver performs the iterative processes to update the extrinsic information between ESE and DESs. After the last iteration, the DESs produce despreading signal.

For the detection of layer- $k$, we can rewrite Eq. (7c) as:

$$
r(j)=h x_{k}(j)+\xi_{k}(j), j=1,2 \ldots, J
$$

where, $\xi_{\mathrm{k}}(\mathrm{j})$, represents the interlayer interference with respect to layer-k.

Similar to[17], for simplicity, our derivation will be based on a single-path channel model with real channel coefficients and BPSK signaling. However, the principle here is not only limited to this case, and can be easy to apply into other cases such as multipath channels, higher order modulations and complex channels[13]-[14].

The ESE function is used to calculate the extrinsic loglikelihood Ratios (LLR) for estimating the transmitted signal. From the definition of the extrinsic LLR, the output of ESE function can be obtained by:

$$
e_{E S E}\left(x_{k}(j)\right)=2 h \cdot \frac{r(j)-E\left(\xi_{k}(j)\right)}{\operatorname{Var}\left(\xi_{k}(j)\right)}
$$

where,

$$
\begin{gathered}
E(r(j))=h \sum_{k} E\left(x_{k}(j)\right) \\
\operatorname{Var}(r(j))=|h|^{2} \sum_{k} \operatorname{Var}\left(x_{k}(j)\right)+\sigma^{2} \\
E\left(\xi_{k}(j)\right)=E(r(j))-h E\left(x_{k}(j)\right) \\
\operatorname{Var}\left(\xi_{k}(j)\right)=\operatorname{Var}(r(j))-|h|^{2} \operatorname{Var}\left(x_{k}(j)\right)
\end{gathered}
$$

The mean and variance of $x_{k}(j)$ can be calculated by the feedback from DESs, as follow:

$$
\begin{aligned}
& E\left(x_{k}(j)\right)=\tanh \left[\frac{e_{D E S}\left(x_{k}(j)\right)}{2}\right] \\
& \operatorname{Var}\left(x_{k}(j)\right)=1-\left(E\left(x_{k}(j)\right)\right)^{2}
\end{aligned}
$$

The mean and variance of the interlayer interference can be used to analyze and detect the signal of each layer. Then the updated extrinsic LLR from ESE function was proven 
to go through the layer-specific de-interleaver and gets into the DESs iteratively[18].

\section{Simulation Results}

In this paper, we carry out the performance analysis of cooperative single user ML-IDMA scheme for equidistant relaying geometry with different number of layers and a MRC technique. The applied relay protocol is Decode-andForward (DF), which is implemented for the system that uses Binary Phase Shift Keying (BPSK). MATLAB is used to simulate the obtained results. In this paper it is assumed that all stations are arranged at the edges of a square with a length of one. That means that all channels will have the same path loss and therefore the same average Signal-toNoise Ratio (SNR).

To simulate the cooperative ML-IDMA scheme, we assume that the channel is Rayleigh fading channel, equidistant relaying geometry, BPSK signaling is used, frame length $(\mathrm{N})=512$, Spreading Length $(\mathrm{SP})=32$, number of relays $(R)=1 \ldots, 4$, number of layers $(K)=2,4$ and number of iteration (it) $=3$.

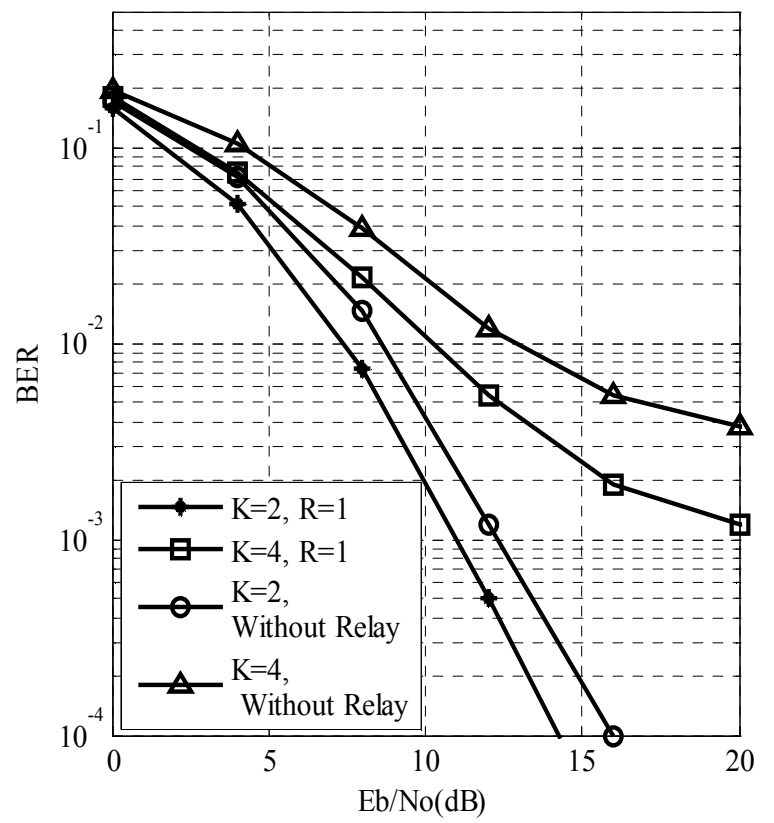

Fig 5. BER of cooperative ML-IDMA in Rayleigh channels with $K=2,4$, $N=512$, $i t=3, S P=32$ and $R=1$

Fig. 5 shows that there is degradation in the BER performance by increasing the number of layers. In the case of without relay, $\mathrm{K}=2$ and $\mathrm{Eb} / \mathrm{N} 0=16 \mathrm{~dB}$, the $\mathrm{BER}$ performance was $10^{-4}$ while increasing $\mathrm{K}$ to 4 , degraded BER performance to $5 * 10^{-3}$. The observed degradation in the BER performance by increasing the number of layers is due to increasing in the signal amplitude which cause signal distortion. For single relay $(\mathrm{R}=1)$, in the case of double layer $(K=2)$, the improvement of the BER performance is $1.7 \mathrm{~dB}$ compared to without relay system. Also in the case of four layer $(\mathrm{K}=4)$ and $\mathrm{Eb} / \mathrm{N0}=20 \mathrm{~dB}$, the BER improvement was found to be about $2.6 * 10^{-3}$ when compared to without relay system as shown in Fig.5.

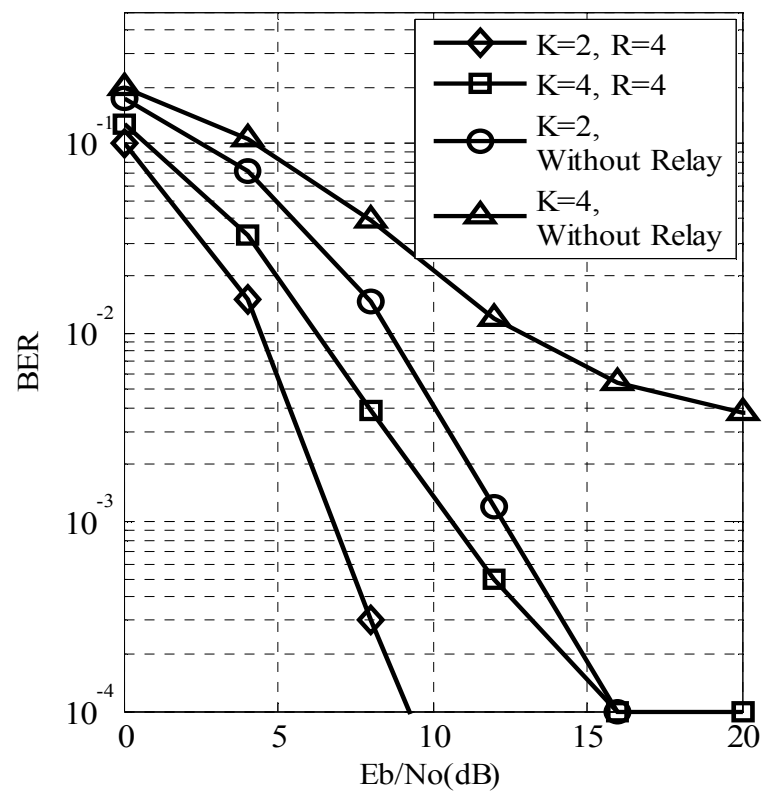

Fig 6. BER of cooperative $M L-I D M A$ in Rayleigh channels with $K=2,4$, $N=512$, $i t=3, S P=32$ and $R=4$

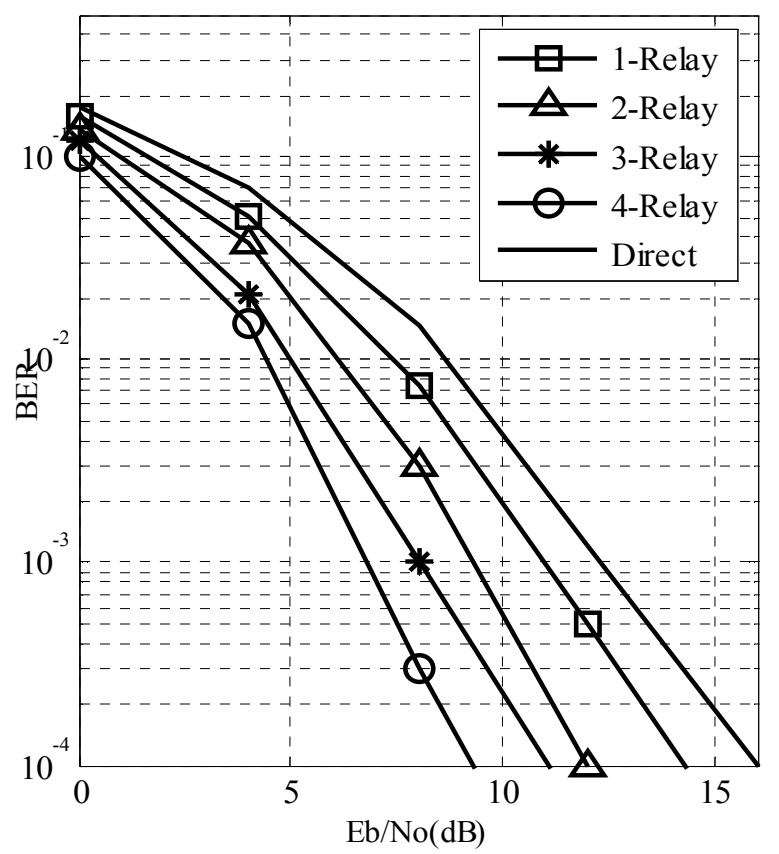

Fig 7. BER of cooperative ML-IDMA system in Rayleigh channels with $R=1,2,3,4, K=2, N=512$, $i t=3$ and $S P=32$

Fig. 6 shows the improvement in BER performance by increasing the number of relays to $\mathrm{R}=4$ at $\mathrm{K}=2$ by about 8 $\mathrm{dB}$ when compared to without relay system. Fig. 7 show that $\mathrm{R}=4$ is superior to the case of $\mathrm{R}=0$ by about $5 \mathrm{~dB}$ and is superior to the case of $\mathrm{R}=1$ by about $6.7 \mathrm{~dB}$ at the $\mathrm{BER}$ of $10^{-4}$. We can conclude that the co-operative environment, which added up two signals of different links, performs better than systems designed without relay environment. We can also see that when additional relay is deployed, the 
performance in the co-operative environment gets better. The analysis showed that the addition of different path powers in co-operative environment results in a lower BitError-Rate (BER)

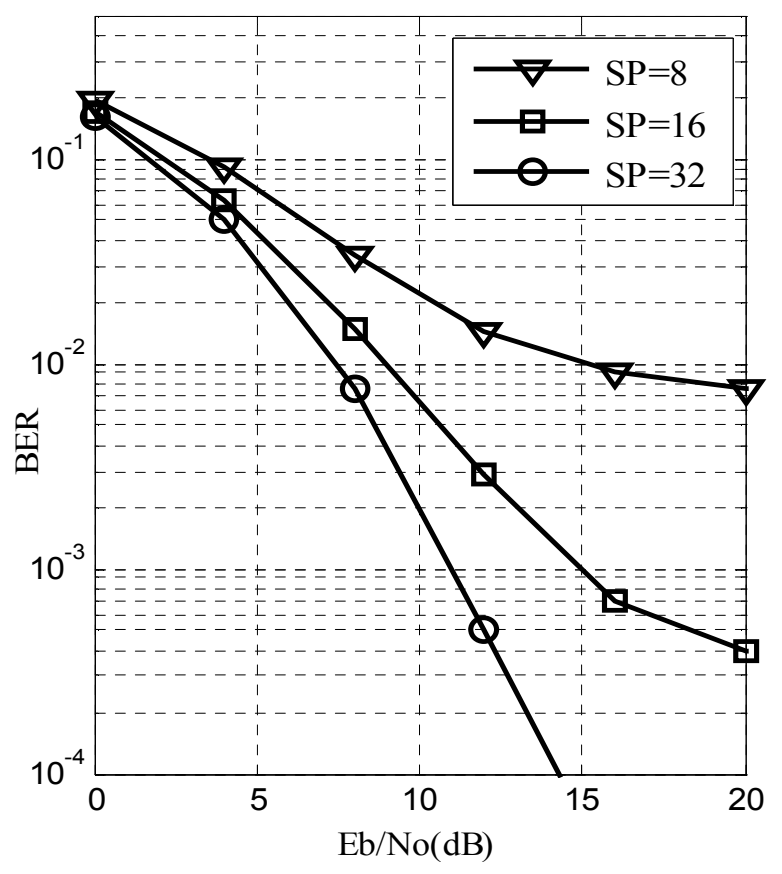

Fig 8. BER of cooperative ML-IDMA system in Rayleigh channels with $S P=8,16,32, R=1, K=2, N=512$ and $i t=3$

Fig. 8 shows that increasing the Spreading Length (SP) can improve the performance significantly. By increasing the SP we were able to get larger spreading gain of the spread spectrum signal that improves the performance efficiency as the signal becomes larger.

\section{Conclusion}

In this paper, a cooperative communication scheme based on ML-IDMA technique is proposed for a network which has one source, one destination and multiple common relays. The proposed system is based on the chipby-chip detection algorithm. Data reaches the destination through two different paths, i.e. a direct path from source to destination and a relayed path, where Decode-and-Forward DF relay protocol is applied to the data that finally reaches the MRC combiner at the destination.

The proposed system has been simulated to evaluate the performance of ML-IDMA technique with different numbers of layers with Maximal-Ratio-Combining (MRC) technique in the cooperative environment. The simulation results showed that by increasing the number of layers, degradation in the performance was obtained but on the other hand the Band Width (BW) was saved by $(1 / \mathrm{K})$.

The simulation also showed that the cooperative environment, which added up two signals of different links, performed better than the system designed without relays. When an additional relay was deployed, the performance in the cooperative environment got better. This improvement means that the addition of different path powers in cooperative environment actually results in a lower Bit Error Rate (BER) in the $\mathrm{E}_{\mathrm{b}} / \mathrm{N}_{0}$ vs. BER curve. We also observed that, the performance of cooperative environment with two or more relays is better in all investigated layers. Future work will be done to investigate the performance of coded ML-IDMA cooperative schemes, OFDM ML-IDMA, Coded OFDM ML-IDMA.

\section{References}

[1] F.Lenkeit, D.Wübben,A.Dekorsy," An Improved Detection Scheme for Distributed IDM-STCs in RelaySystems ",IEEE 76th Vehicular Technology Conference (VTC2012-Fall), Québec, Canada, 3. - 6. September 2012.

[2] J. N. Laneman, and G. W. Wornell, "Distributed SpaceTime-Coded Protocols for Exploiting Cooperative Diversity in Wireless Networks," IEEE Trans. Inf. Theory, vo.49, no.10, pp.2415-2425, Oct 2004.

[3] G. Scutari, S. Barbarossa," Distributed space-time coding for regenerative relay networks," IEEE Trans. Wireless Commun., vol.4, no.5, pp.2387-2399, Sept. 2005.

[4] Jinhong Yuan, Yonghui $\mathrm{Li}$, and $\mathrm{Li} \mathrm{Chu}$, "Differential Modulation and Relay Selection WithDetect-and-Forward Cooperative Relaying," IEEE Trans. ON Vehicular Technology, vol. 59, no. 1, jan. 2010.

[5] L. Ping, L. Liu, K. Wu, and W. K. Leung, "Interleavedivision multipleaccess," IEEE Trans. Wireless Commun., vol. 5, no. 4, pp. 938-947, Apr. 2006.

[6] Zhifeng Luo, Deniz Gurkan, Zhu Han, Albert Kai-sun Wong and Shuisheng Qiu," Cooperative Communication Based on IDMA," 5th International Conference on Wireless Communications, Networking and Mobile Computing, pp.1-4, Sep. 2009.

[7] J. Tong, P. Li, Z. H. Zhang, and W. K. Bhargava, "Iterative Soft Compensation for OFDM Systems with Clipping and Superposition Coded Modulation," IEEE Trans. Commun., vol. 58, no. 10, pp. 2861-2870, Oct. 2010.

[8] Zhifeng Luo, Deniz Gurkan, Zhu Han, Albert Kai-sun Wong and Shuisheng Qiu,"Cooperative Communication Based on IDMA," 5th International Conference on Wireless Communications, Networking and Mobile Computing, pp.1-4, Sep. 2009.

[9] Xiaotian Zhou, Haixia Zhang and Dongfeng Yuan,"A multi-source cooperative scheme based on IDMA aided superposition modulation," International Conference on Information Theory and Information Security, pp.802-806, December 2010.

[10] Weitkemper, P. , Wubben, D. and Kammeyer, K.-D.," Delay-diversity in multi-user relay systems with Interleave Division Multiple Access," IEEE International Symposium on Wireless Communication Systems 2008, pp.364-368, October 2008 .

[11] Chulhee Jang and Jae Hong Lee, "Novel IDM-cooperative diversity scheme and power allocation," in Proc. IEEE APWCS 2009, Seoul, Korea, pp.24-28, Aug. 2009. 
[12] J. N. Laneman, D. N. C. Tse and G. W. Wornell, "Cooperative Diversity In Wireless Networks: Efficient Protocols And Outage Behavior", IEEE Trans. Info. Theory, Vol. 50, No. 12, pp. 3062-3080, November 2004.

[13] P. Li, L. H. Liu, K. Y. Wu, and W. K. Leung, "Interleave division multiple access," IEEE Trans. Wireless Commun., vol. 5, no. 4, pp. 938-947, Apr. 2006.

[14] P. Li, Q. H. Guo, and J. Tong, "The OFDM-IDMA approach to wireless communication systems," IEEE Wireless Commun. Mag., vol. 14, no. 3, pp. 18-24, Jun. 2007.

[15] Takyu O, Ohtsuki T, Nakagawa M. Companding system based on time clustering for reducing peak power of OFDM symbol in wireless communication. IEICE Transactions on Fundamentals of Electronics Communications and Computer Sciences 2006; E89- A(7): 1884-91.
[16] J. Akhtman, B. Z. Bobrovsky, and L. Hanzo, "Peak-toaverage power ratio reduction for ODFM modems," in Proceedings of the 57th IEEE Semi-Annual Vehicular Technology Conference (VTC'03), vol. 2, Apr. 2003, pp. $1188-1192$.

[17] P. Li, L. H. Liu, K. Y. Wu, and W. K. Leung, "A unified approach to multiuser detection and space-time coding with low complexity and nearly optimal performance," in Proc. 40th Allerton Conference, Allerton House, USA, pp. 170 179, Oct. 2002.

[18] Tao Peng, and Min Ye, "PAPR mitigation in superposition coded modulation systems using selective mapping" in Proceeding of the International Conference on Computer and Communication Technologies (ICCCT'2012), May 2627, 2012, Phuket. 